Case Report

\title{
Pneumococcal Meningitis Complicated by Cerebral Vasculitis, Abscess, Hydrocephalus, and Hearing Loss
}

\author{
Abdul Razzakh Poil $\mathbb{D}^{1}{ }^{1}$ Adila Shaukat, ${ }^{1}$ and Devendra Kumar ${ }^{2}$ \\ ${ }^{1}$ Consultant, Medicine, Hamad Medical Corporation, Doha, Qatar \\ ${ }^{2}$ Consultant, Radiology, Hamad Medical Corporation, Doha, Qatar \\ Correspondence should be addressed to Abdul Razzakh Poil; drrazzakh@gmail.com \\ Received 18 February 2018; Accepted 16 September 2018; Published 16 October 2018 \\ Academic Editor: Paola Di Carlo \\ Copyright (c) 2018 Abdul Razzakh Poil et al. This is an open access article distributed under the Creative Commons Attribution \\ License, which permits unrestricted use, distribution, and reproduction in any medium, provided the original work is \\ properly cited.

\begin{abstract}
Intracranial abscesses, postinfectious vasculitis, and hydrocephalus are rare complications of Streptococcus pneumoniae (S. pneumoniae) meningitis, and to our knowledge, there have been no case reports where all these 3 complications occurred in a single patient with Streptococcus pneumoniae meningitis. Here, we report a case of a 48-year-old male who developed postinfectious vasculitis, abscess, hydrocephalus, and hearing loss after $S$. pneumoniae meningitis. Clinicians ought to be aware of the possible adverse outcomes of $S$. pneumoniae meningitis and the limitations of current treatment options.
\end{abstract}

\section{Introduction}

The mortality rate among patients with bacterial meningitis and the frequency of neurological sequelae among those who survive are high, despite major progress in intensive care and effective antimicrobial chemotherapy. Postinfectious vasculitis leading to ischemic brain damage is rare, but a known complication of bacterial meningitis and its treatment are uncertain [1-3]. The mortality rate is higher among patients with pneumococcal meningitis than among those with meningococcal meningitis [4]. This increased mortality and morbidity are due to high neurological (74.7\%) and systemic (37.9\%) complications. Seizure (27.6\%), diffuse brain swelling (28.7\%), hydrocephalus (16.1\%), hearing loss (19.7\%), and ischemic or hemorrhagic brain damage (21.8\%) are some of the complications [5].

Streptococcus pneumoniae intracranial abscess is a rare complication of pneumococcal meningitis, with the majority of cases being reported in the preantibiotic era. Today, the survival rate and long-term outcome of these patients remain quite poor, with up to $35 \%$ mortality and $40 \%$ experiencing prolonged neurological deficits [6-8]. Intracranial abscesses, postinfectious vasculitis, and hydrocephalus are rare complications of $S$. pneumoniae meningitis, and to our knowledge, there have been no case reports where all these 3 complications occurred in a single patient with Streptococcus pneumoniae meningitis. There was a case report of postinfectious vasculitis and abscess of brain following Streptococcus pneumoniae meningitis [6]. Here, we report a case of a 48 -year-old male with $S$. pneumoniae meningitis complicated by postinfectious cerebral vasculitis, intracranial abscess, hydrocephalus, hearing loss, and brain infarct.

\section{Case Report}

A 48-year-old Indian male with no chronic medical illness in the past admitted to emergency department with history of fever, headache, dry cough, and generalized body pain for 4 days and vomiting for one day. No abdominal pain, SOB, chest pain, joint pain, skin rash, recent travel, or exposure to sick person and no significant family history were reported. Patient denied alcohol consumption or tobacco smoking. On physical examination, the patient was well built and nourished; he was icteric, conscious, and oriented to time, place, and person. Vitals were as follows: temperature: 
afebrile, $35.9^{\circ} \mathrm{C}$; heart rate: $94 /$ minute; respiratory rate: 20/minute; blood pressure: $121 / 81 \mathrm{mmHg}$; and $\mathrm{SpO}_{2}$ : $98 \%$ in room air.

Systemic examination showed normal neurological findings except meningeal signs. Other systems were unremarkable. Initial investigations showed hemoglobin and platelets were normal. White blood cell (WBC) count was $12.6 \times 10^{3} /$ microliter (normal: $4 \times 10^{3} /$ microliter-10 $\times$ $10^{3} /$ microliter) with $92 \%$ neutrophils. Serum creatinine was $146 \mu \mathrm{mol}$ per liter (normal: 64 to $110 \mu \mathrm{mol}$ per liter), urea was $11 \mathrm{mmol}$ per liter (normal: $3.2 \mathrm{mmol}$ per liter to $7.4 \mathrm{mmol}$ per liter), and serum electrolytes were normal. Alanine aminotransferase (ALT) was 56 units per liter (normal: 0 units per liter to 30 units per liter), aspartate aminotransferase (AST) was 38 units per liter (normal: 0 units per liter to 31 units per liter), alkaline phosphatase (ALP) was 96 units per liter (normal: 40 units per liter to 150 units per liter), albumin was $33 \mathrm{~g}$ per liter (normal: $35 \mathrm{~g} / \mathrm{L}$ to $50 \mathrm{~g} / \mathrm{L}$ ), total bilirubin was $68 \mu \mathrm{mol}$ per liter (normal: 3.4 to $20.5 \mu \mathrm{mol}$ per liter), direct bilirubin was $34 \mu \mathrm{mol}$ per liter (normal: 0 to $8.6 \mu \mathrm{mol}$ per liter), C-reactive protein (CRP) was $495 \mathrm{mg}$ per liter (normal: 0 to $5 \mathrm{mg} / \mathrm{L}$ ), procalcitonin (PCTN) was $11 \mathrm{ng}$ per milliliter (normal: 0 to $2 \mathrm{ng} / \mathrm{mL}$ ), and chest X-ray was normal. His conscious level deteriorated soon after hospital admission, and the Glasgow Coma Scale (GCS) dropped from $15 / 15$ to $12 / 15$. Meningitis is suspected, and antibiotics were started after lumbar puncture (LP) and computerized tomography (CT) head. Initial empirical intravenous (IV) antibiotics were started: ceftriaxone, vancomycin, and acyclovir along with dexamethasone. CT head was normal, and cerebrospinal fluid (CSF) analysis showed WBC 145 per microliter (normal: 0 to $5 / \mu \mathrm{L}$ ) with $96 \%$ neutrophils, protein 4.55 grams per liter (normal: 0.15 to $0.45 \mathrm{~g} / \mathrm{L}$ ), and Glu $<0.3 \mathrm{mmol}$ per liter; CSF viral panel showed Epstein-Barr virus (EBV) PCR was 326 International Units per $\mathrm{mL}$, acid-fast bacilli staining was negative, and tuberculosis PCR was negative. Both CSF and blood culture showed Streptococcus pneumonia which was sensitive to ceftriaxone. So acyclovir and vancomycin were stopped, dexamethasone was given for a total of 3 days, and IV ceftriaxone $2 \mathrm{~g}$ every 12 hours was continued; later, the patient's condition improved and oriented to person but not oriented to time and place. There was no focal neurological deficit. However, he developed hard of hearing and though fever pattern has improved, having fever spike on and off. Repeat blood cultures were negative, CRP improved from $>500 \mathrm{mg} / \mathrm{L}$ to $93 \mathrm{mg} / \mathrm{L}$, PCTN decreased from $11 \mathrm{ng} / \mathrm{mL}$ to $0.41 \mathrm{ng} / \mathrm{mL}$, and serum creatinine level normalized. However, liver enzymes persisted to rise (both transaminases and alkaline phosphatase), and white blood cell persisted to be in the range of 13-15. Due to presence of persistence of symptoms, magnetic resonance image (MRI) brain and repeat LP were done to rule out any complication of the disease. MRI brain (Figures 1 and 2) showed meningoencephalitis, vasculitis, and extradural fluid collection. There was right-sided fluid in the mastoid cavity without bone destruction. ENT was consulted and advised for medical management. As the patient is confused, hearing assessment could not be done properly. Repeat LP showed CSF WBC $11 / \mu \mathrm{L}$, neutrophils $70 \%$, lymphocytes $29 \%$, protein $1.11 \mathrm{~g} / \mathrm{L}$, EBV PCR was negative, and CSF culture was negative. Dexamethasone was restarted with continuation of IV ceftriaxone for a total of 6 weeks as Streptococcus pneumoniae meningitis is complicated by infective vasculitis, mastoiditis, and subdural collection. Repeat MRI brain (Figure 3) showed significant improvement in leptomeningeal enhancement and resolution of epidural collection; however, there was a new communicating hydrocephalus. After completion of his IV ceftriaxone, the patient was repatriated to his home country. Although his condition improved on above treatment, he was discharged with mild disorientation to time and person.

\section{Discussion}

This complex case highlights the risk of intracranial complications in invasive pneumococcal infections. Invasive pneumococcal disease is defined as an infection caused by Streptococcus pneumoniae isolated from a normally sterile site such as blood, cerebrospinal fluid, and pleural, joint, or peritoneal fluid. The incidence of invasive pneumococcal disease in the USA was 36.4 cases per 100,000 population above the age of 65 years, 34.2 cases per 100,000 population in infants below 1 year, and 3.8 cases per 100,000 population in the age between 18 and 34 years [9]. Data about the prevalence of invasive pneumococcal disease in Qatar are lacking. According to one report, the incidence of the disease in the general Arab region was $7 \%$ above the age of 60 years, and this is expected to increase $19 \%$ by 2050 ; however, incidents in Qatar population are high than those in the general Arab population (20.7\%) which are comparable to those in population in the USA (26.9\%) [10]. Since extremes of ages are predisposing conditions towards invasive pneumococcal disease, an effective vaccination program is very important to decrease the morbidity and mortality due to this condition. Despite effective antibiotic treatment, mortality and morbidity are high in patients with bacterial meningitis. Morbidity like vasculitis after bacterial meningitis which can lead to brain injury is one of the known complications, but its treatment is unclear [1-3]. Among bacterial meningitis, the most common organisms were Streptococcus pneumoniae and Neisseria meningitidis. The mortality rate was significantly higher with pneumococcal meningitis $(30 \%)$ when compared with meningococcal meningitis (7\%) [4]. S. pneumoniae causes alteration in the host inflammatory response and results in the release of proinflammatory cytokines in the cerebrospinal fluid (CSF) which results in disruption of blood-brain barrier (BBB) [11]. This results in increased neurological (74.7\%) and systemic (37.9\%) complications. These include seizure $(27.6 \%)$, diffuse brain swelling $(28.7 \%)$, hydrocephalus $(16.1 \%)$, hearing loss (19.7\%), and ischemic or hemorrhagic brain damage (21.8\%) [5]. However, vasculitis is not a common sequela of meningitis caused by $S$. pneumoniae 


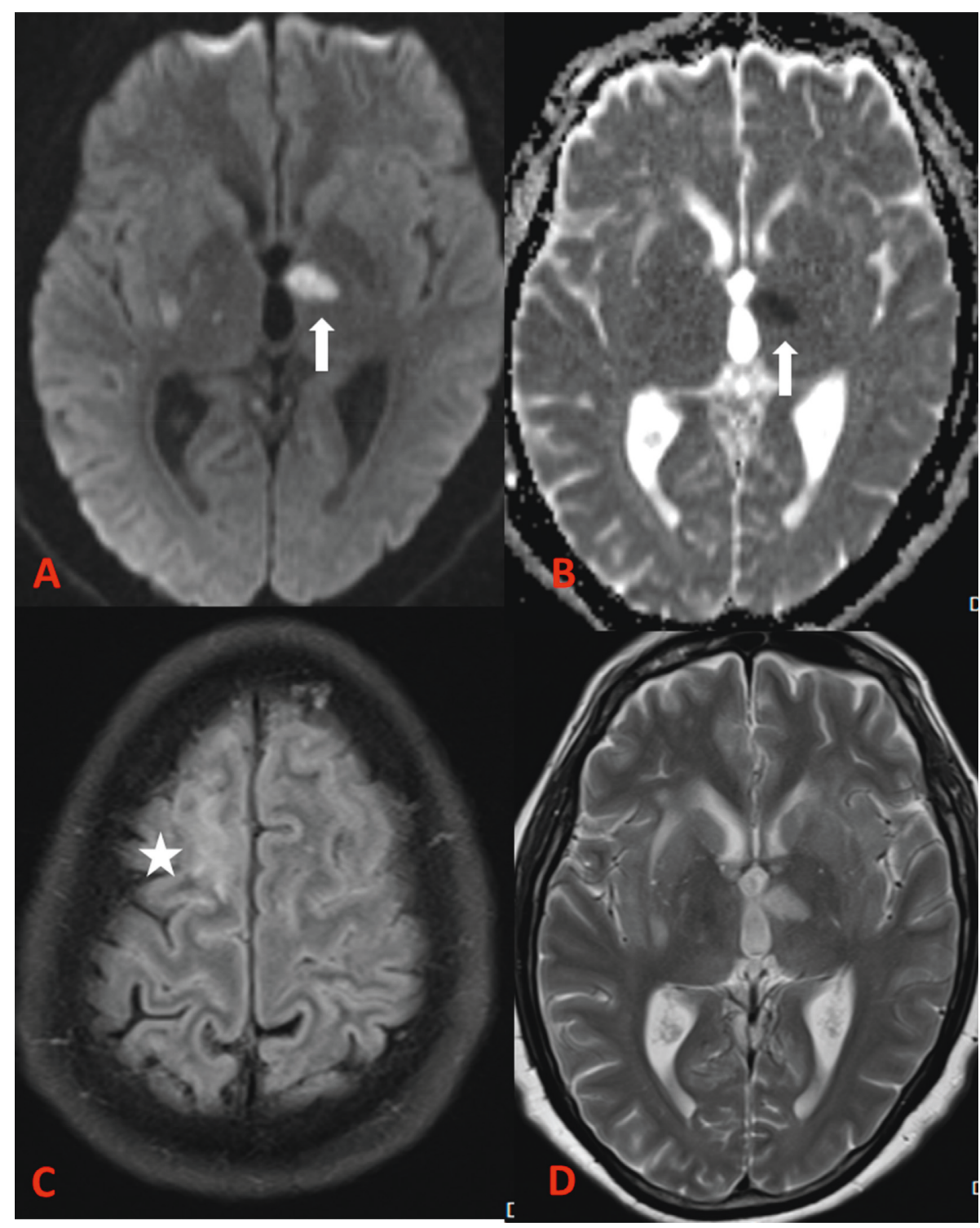

FIGURE 1: MRI brain examination of a 48-year-old male with meningoencephalitis, vasculitis, and extradural fluid collection. Axial DWI (A) and ADC (B) images show acute left thalamic (white arrow) and tiny right basal ganglia lacunar infarcts secondary to vasculitis. Axial FLAIR image (C) shows a cortical high signal in bilateral higher parietal lobes suggestive of epidural collection and underlying cerebritis (star). Axial T2-weighted image (D) shows a hyperintense signal in the left thalamus and basal ganglia secondary to vasculitis.

[5]. Despite advancement in the medical field, only dexamethasone has shown to be effective adjunctive therapy. It lowers the rate of hearing loss and mortality [12]. Pneumococcal meningitis is associated with $54 \%$ of hearing loss (69\% in adults and $31 \%$ in children). Advanced age, presence of comorbidity, severity of meningitis, a low CSF glucose level, and a high CSF protein level are the risk factors for hearing loss [13].

Although intracranial abscess after Streptococcus pneumoniae meningitis is a rare complication, the survival rate and long-term outcome are very poor, with up to $35 \%$ mortality and $40 \%$ long-term neurological sequelae [6-8].

Risk of complications in bacterial meningitis is higher in patients with advanced age, presence of otitis or sinusitis, absence of rash, a low score on the Glasgow Coma Scale, tachycardia, a positive blood culture, an elevated erythrocyte sedimentation rate, thrombocytopenia, and a low cerebrospinal fluid white-cell count [4]. Our patient has a higher risk of invasive S. pneumoniae. The majority of patients have an obvious secondary cause such as ear or sinus infections or pneumonia $[5,6]$.

Several studies have reported that hydrocephalus may occur in adults as well as in children with bacterial meningitis. The most common causative agent is S. pneumoniae. Among adults, the reported incidence of this complication ranged from 3 to $21 \%$. In spite of surgical intervention, prognosis is poor with the mortality rate up to $50 \%$ [14-16]. 


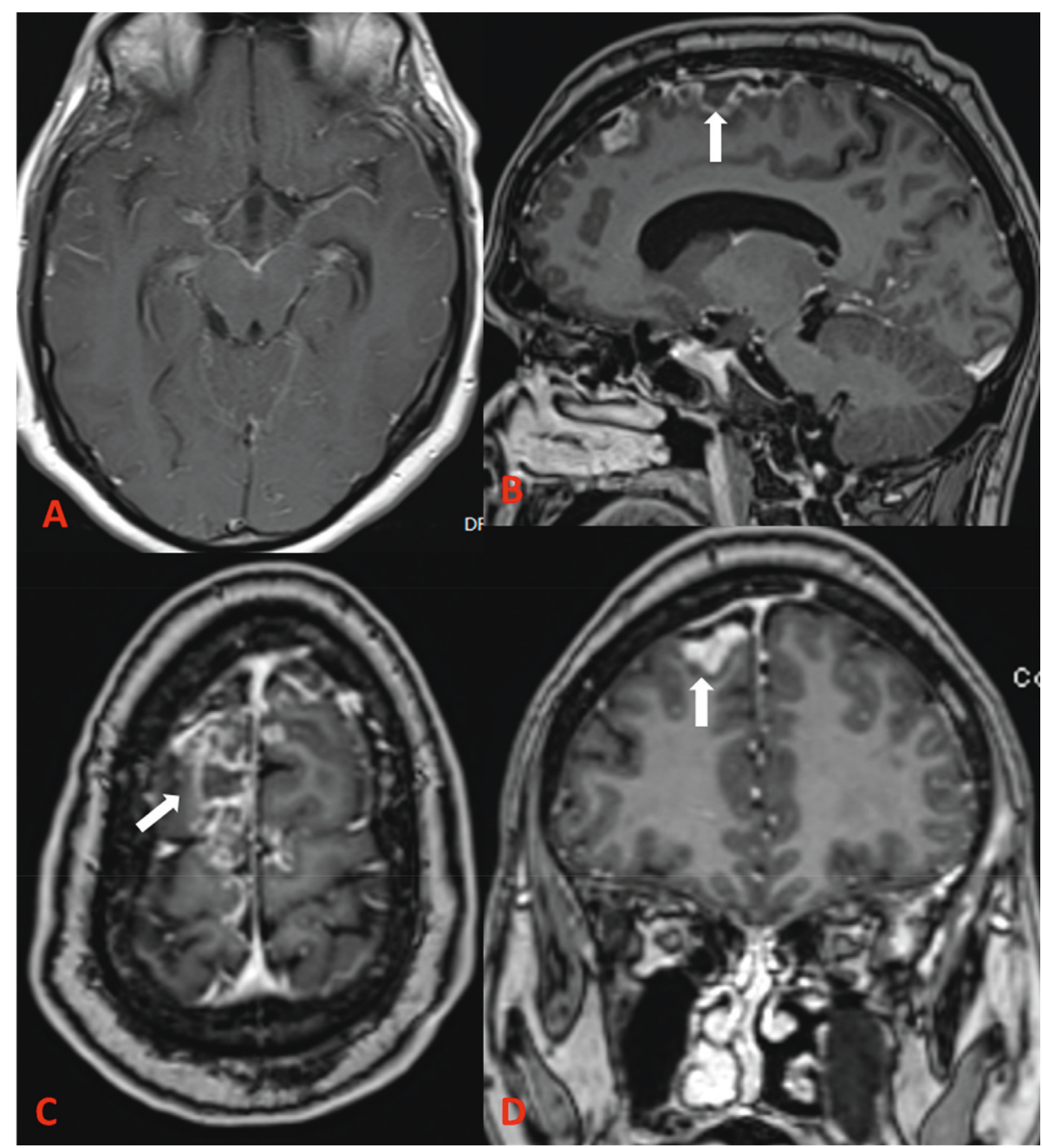

FIgURE 2: MRI brain examination of a 48-year-old male with meningoencephalitis, vasculitis, and extradural fluid collection. Postcontrast MRI axial T1 (A), GRE sagittal (B), GRE axial (C), and GRE coronal (D) images show diffuse leptomeningeal enhancement, enhancing basal exudates and high biparietal epidural fluid collection (white arrow) with cerebritis.

Given the rarity of postmeningitis complications like cerebral vasculitis, sensorineural hearing loss, and hydrocephalus, there is no consensus on treatment for these conditions. It has been shown that treatment with dexamethasone in patients with pneumococcal meningitis can decrease the complication, including postinfectious vasculitis and mortality [12]. Corticosteroids have been recommended as adjunctive therapy for bacterial meningitis to reduce the complications like hearing loss especially when given early during the disease course [13, 17]. Although treatment is usually given less than four days, some reports have shown a rebound vascular inflammation after steroid withdrawal [18]. Dexamethasone therapy was shown to decrease the incidence of hydrocephalus [16]. Due to these growing bodies of the literature, we have treated our patient with a prolonged taper of steroids.

This patient's immunization status was not known; however, his immune system seems normal. He was not on any immunosuppressive medications, and he had no chronic medical illness. He did not have leukopenia, and his HIV test was negative. Hepatitis $\mathrm{C}$ antibody, hepatitis B surface antibody, and hepatitis B surface antigen were negative. Invasive pneumococcal disease is a vaccine-preventable disease. Currently, there are 2 vaccines available: conjugate vaccine (PCV13) and polysaccharide vaccine PPSV23 [19]. Since the introduction of conjugate vaccine in children, a gradual decrease has been noticed in the incidence of invasive pneumococcal disease both in vaccinated and nonvaccinated population most likely due to herd immunity [20-23]. But it has also been observed that pneumococcal infections due to pneumococcal serotypes that are not included in the vaccine have been rising. As per current recommendation, a dose of PCV13 has to be given followed by a dose of PPSV 23 in all adults aged $\geq 65$ years who did not receive pneumococcal vaccine before and in persons aged $\geq 2$ years who are at high risk for pneumococcal disease like immunocompromised patients, functional or anatomic asplenia, cochlear implants, or cerebrospinal fluid leaks [24]. In Qatar, in healthy adults over 50 years, a single dose of PCV13 is given 5 years from last PPV23; additionally, high-risk adults 


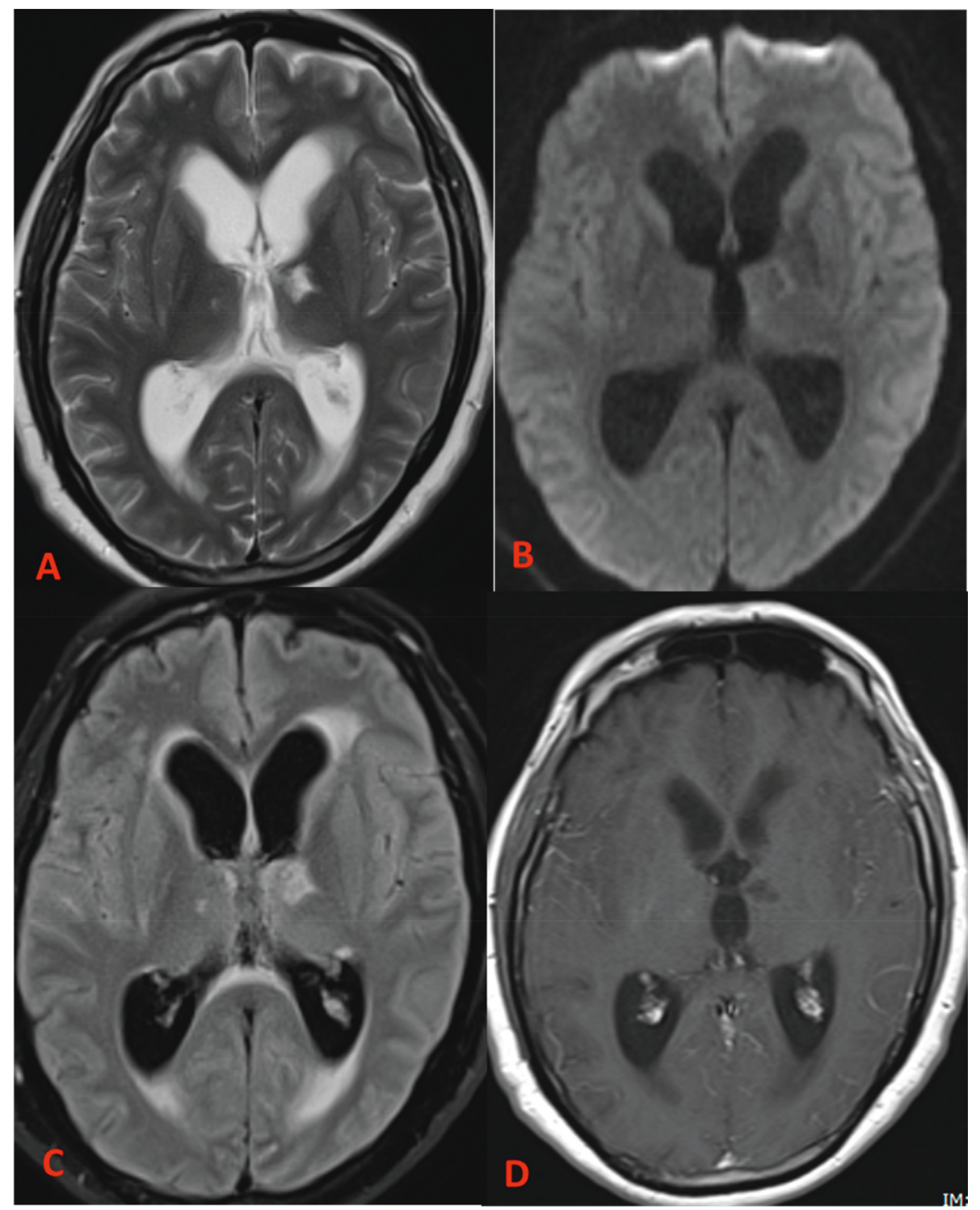

FIGURE 3: Follow-up MRI brain examination after one month. Axial T2 (A), DWI (B), FLAIR (C), and postcontrast T1-weighted (D) images show significant interval improved leptomeningeal enhancement, resolution of epidural collection (not shown), and interval new communicating hydrocephalus.

aged 19-64 years are administered a single dose of PCV13 at a yearly interval from the last dose of PPV23, and adults who have not been previously vaccinated are administered a single dose of PCV13, followed by PPV23 eight weeks later [25].

\section{Conflicts of Interest}

The authors declare that there are no conflicts of interest regarding the publication of this paper.

\section{Authors' Contributions}

Abdul Razzakh Poil is the principal investigator. Adila Shaukat and Devendra Kumar are coinvestigators.

\section{References}

[1] D. Pugin, J.-C. Copin, M.-C. Goodyear, T. Landis, and Y. Gasche, "Persisting vasculitis after pneumococcal meningitis," Neurocritical Care, vol. 4, no. 3, pp. 237-240, 2006.

[2] M. L. Durand, S. B. Calderwood, D. J. Weber et al., "Acute bacterial meningitis in adults. A review of 493 episodes," New England Journal of Medicine, vol. 328, no. 1, pp. 21-28, 1993.

[3] H. W. Pfister, W. Feiden, and K. M. Einhaupl, "Spectrum of complications during bacterial meningitis in adults. Results of a prospective clinical study," Archives of Neurology, vol. 50, no. 6, pp. 575-581, 1993.

[4] D. van de Beek, J. de Gans, L. Spanjaard, M. Weisfelt, J. B. Reitsma, and M. Vermeulen, "Clinical features and 
prognostic factors in adults with bacterial meningitis," New England Journal of Medicine, vol. 351, no. 18, p. 1849, 2004.

[5] S. Kastenbauer and H. W. Pfister, "Pneumococcal meningitis in adults: spectrum of complications and prognostic factors in a series of 87 cases," Brain, vol. 126, no. 5, pp. 1015-1025, 2003.

[6] A. Lucas, K. K. Maung, and R. Ryan, "Streptococcus pneumoniae intracranial abscess and post-infectious vasculitis," Infectious Disease Reports, vol. 8, no. 4, p. 6844, 2016.

[7] E. Grigoriadis and W. L. Gold, "Pyogenic brain abscess caused by streptococcus pneumonia: case report," Clinical Infectious Diseases, vol. 25, no. 5, pp. 1108-1112, 1997.

[8] J. H. Tseng and M. Y. Tseng, "Brain abscess in 142 patients: factors influencing outcome and mortality," Surgical Neurology, vol. 65, no. 6, pp. 557-562, 2006.

[9] Centers for Disease Control and Prevention, Active Bacterial Core Surveillance (ABCs) Report Emerging Infections Program Network: Streptococcus pneumoniae, 2010, http://www.cdc. gov/abcs/reports-findings/survreports/spneu10-orig.pdf.

[10] B. MirkinArab, Human Development Report-Population Levels, Trends and Policies in the Arab Region: Challenges and Opportunities, United Nations Development Series, New York, NY, USA, 2012.

[11] M. G. Tauber and B. Moser, "Cytokines and chemokines in meningeal inflammation: biology and clinical implications," Clinical Infectious Diseases, vol. 28, no. 1, pp. 1-11, 1999.

[12] J. de Gans and D. van de Beek, "Dexamethasone in adults with bacterial meningitis," New England Journal of Medicine, vol. 347, no. 20, pp. 1549-1556, 2002.

[13] L. Worsøe, P. Caye-Thomasen, C. T. Brandt, J. Thomsen, and C. Østergaard, "Factors associated with the occurrence of hearing loss after pneumococcal meningitis," Clinical Infectious Diseases, vol. 51, no. 8, pp. 917-924, 2010.

[14] E. S. Kasanmoentalib, M. C. Brouwer, A. van der Ende, and D. van de Beek, "Hydrocephalus in adults with communityacquired bacterial meningitis," Neurology, vol. 75, no. 10, pp. 918-923, 2010.

[15] K. W. Wang, W. N. Chang, H. W. Chang, H. C. Wang, and C. H. Lu, "Clinical relevance of hydrocephalus in bacterial meningitis in adults," Surgical Neurology, vol. 64, no. 1, pp. 61-65, 2005.

[16] J. Bodilsen, H. C. Schønheyder, and H. Nielsen, "Hydrocephalus is a rare outcome in community-acquired bacterial meningitis in adults: a retrospective analysis," BMC Infectious Diseases, vol. 13, no. 1, p. 321, 2013.

[17] D. van de Beek, J. J. Farrar, J. de Gans et al., "Adjunctive dexamethasone in bacterial meningitis: a meta-analysis of individual patient data," Lancet Neurology, vol. 9, no. 3, pp. 254-263, 2010.

[18] S. Ribeiro, V. Domingues, R. M. Faria, and T. Mendonça, "Invasive pneumococcal disease complicated by cerebral vasculitis, transient diabetes insipidus and spondylodiscitis," BMJ Case Reports, vol. 2013, article bcr2013010336, 2013.

[19] Centers for Disease Control and Prevention (CDC), "Use of 13 -valent pneumococcal conjugate vaccine and 23-valent pneumococcal polysaccharide vaccine for adults with immunocompromising conditions: recommendations of the Advisory Committee on Immunization Practices (ACIP)," Morbidity and Mortality Weekly Report, vol. 61, p. 816, 2012.

[20] T. R. Talbot, K. A. Poehling, T. V. Hartert et al., "Reduction in high rates of antibiotic-nonsusceptible invasive pneumococcal disease in Tennessee after introduction of the pneumococcal conjugate vaccine," Clinical Infectious Diseases, vol. 39, no. 5, pp. 641-648, 2004.
[21] Centers for Disease Control and Prevention (CDC), "Direct and indirect effects of routine vaccination of children with 7valent pneumococcal conjugate vaccine on incidence of invasive pneumococcal disease--United States, 1998-2003," Morbidity and Mortality Weekly Report, vol. 54, p. 893, 2005.

[22] S. S. Shah and A. J. Ratner, "Trends in invasive pneumococcal disease-associated hospitalizations," Clinical Infectious Diseases, vol. 42, no. 1, pp. e1-e5, 2006.

[23] C. A. Lexau, R. Lynfield, R. Danila et al., "Changing epidemiology of invasive pneumococcal disease among older adults in the era of pediatric pneumococcal conjugate vaccine," JAMA, vol. 294, no. 16, p. 2043, 2005.

[24] M. Kobayashi, N. M. Bennett, R. Gierke et al., "Intervals between PCV13 and PPSV23 vaccines: recommendations of the advisory committee on immunization practices (ACIP)," MMWR. Morbidity and Mortality Weekly Report, vol. 64, no. 34, pp. 944-947, 2015.

[25] C. Feldman, E. Abdulkarim, F. Alattar et al., "Pneumococcal disease in the Arabian Gulf: recognizing the challenge and moving toward a solution," Journal of Infection and Public Health, vol. 6, no. 6, pp. 401-409, 2013. 


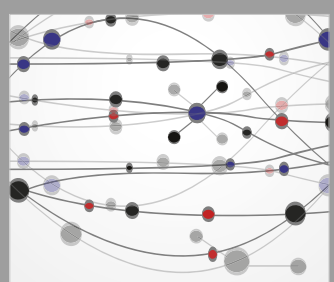

The Scientific World Journal
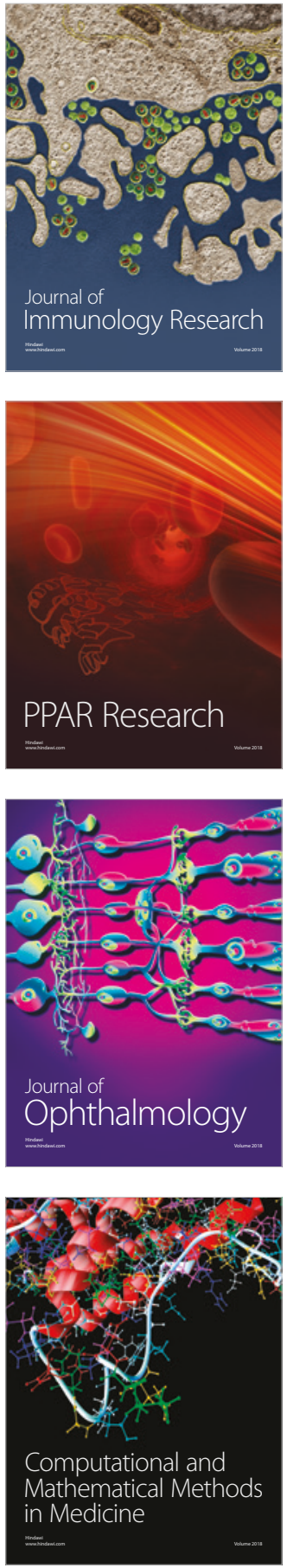

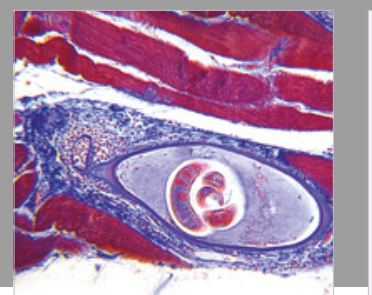

Gastroenterology Research and Practice

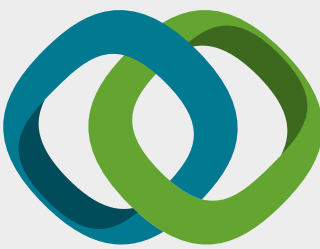

\section{Hindawi}

Submit your manuscripts at

www.hindawi.com
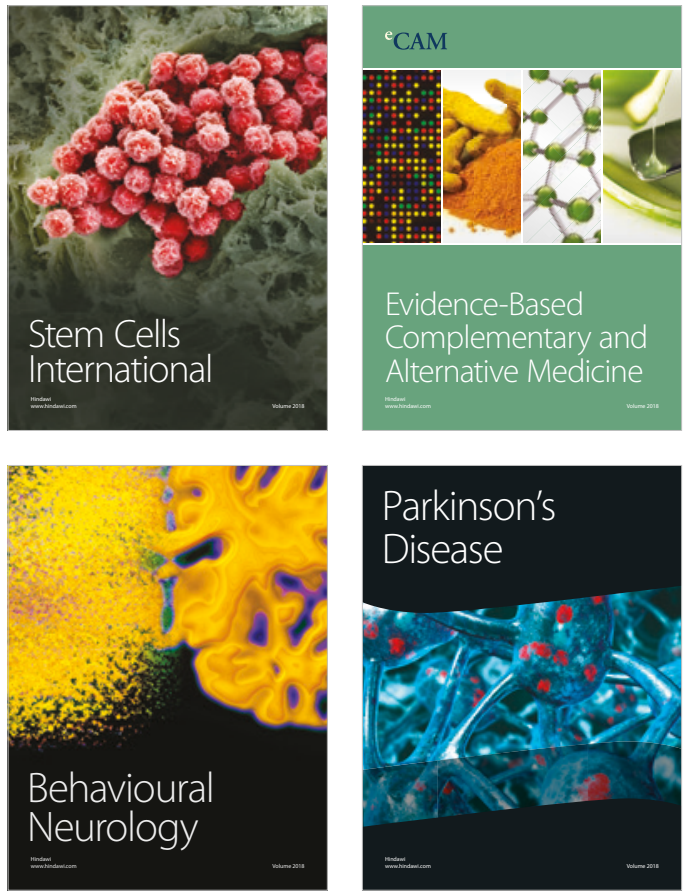

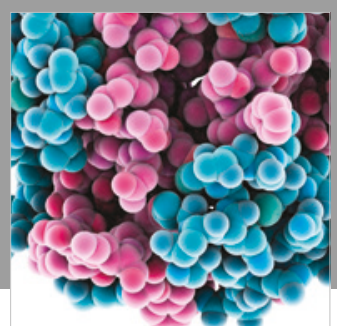

ournal of

Diabetes Research

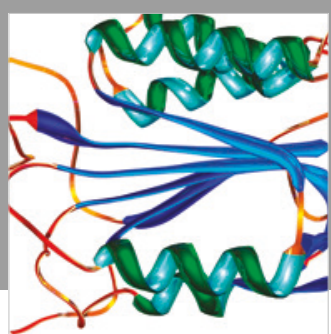

Disease Markers
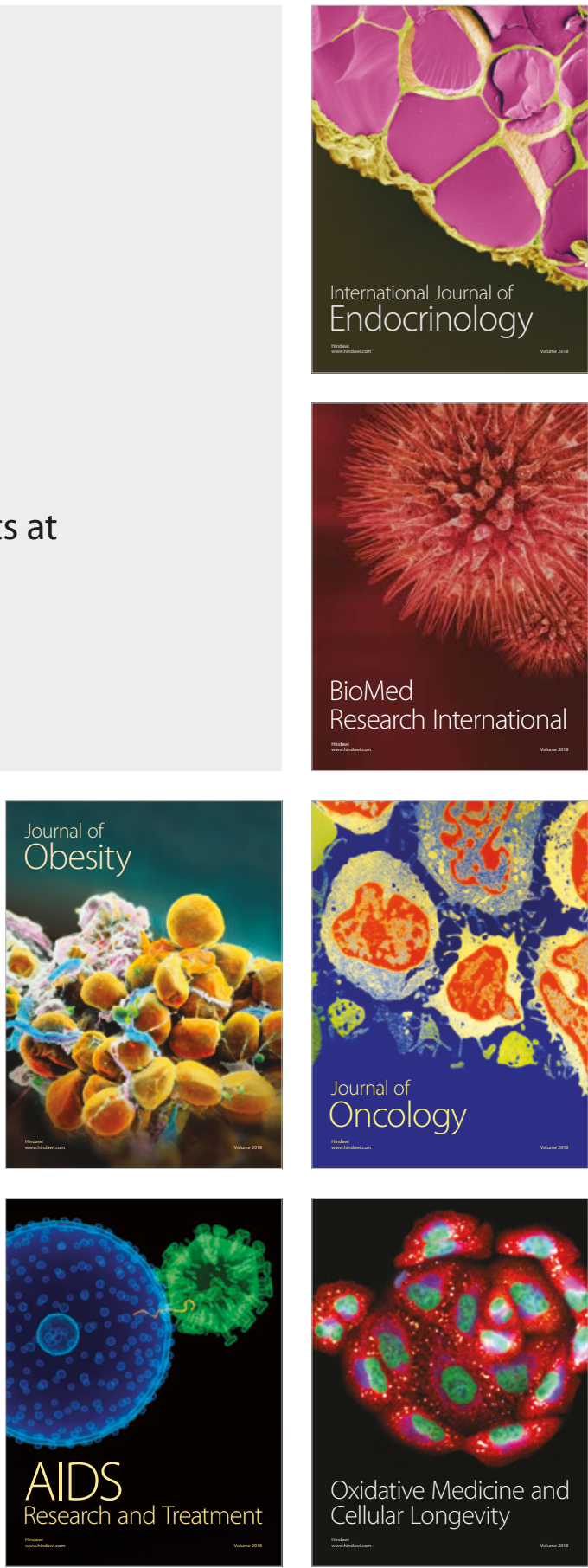